\title{
Organ Damage due to Elevation of Blood Pressure on NaCl-induced
}

\author{
Hotimah Masdan Salim ${ }^{*}$, Ilham Putra Alam², Widya Dio Kharisma² \\ ${ }^{1}$ Department of Medical Biochemistry, Faculty of Medicine, Universitas Nahdlatul Ulama, Surabaya, Indonesia \\ ${ }^{2}$ Faculty of Medicine, Universitas Nahdlatul Ulama, Surabaya, Indonesia
}

\section{A R T I C L E I N F O}

\section{Article history:}

Received 17 September 2020

Received in revised form 5

October 2020

Accepted 29 October 2020

Available online 31 October 2020

Keywords:

$\mathrm{NaCl}$-induce,

Hypertension,

Organs.

*) Corresponding author:

dr.hotimah@unusa.ac.id

\begin{abstract}
A B S T R A C T
Introduction: High salt diet is known to induce or aggravate hypertension in hypertensive rats and humans. The elevation of blood pressure by $\mathrm{NaCl}$-induced promotes cardiac hypertrophy, the impairment of left ventricular relaxation, endothelial dysfunction, and kidney injury. This study aimed to examine whether $\mathrm{NaCl}$-induced caused increase blood pressure and weight of organs.

Methods: Eight weeks old male Mus musculus were divided to two groups, one group was given $\mathrm{NaCl} 8 \%$ by intraperitoneal injection for 8 hours.

Results: Blood pressure was measured previously, in this study systolic and diastolic blood pressure increased significantly $(\mathrm{p}<0.05)$. The increasing of blood pressure was followed by significant increase in organ weight, such as heart and kidney $(\mathrm{p}<0.05)$.

Conclusion: This result suggested that $\mathrm{NaCl}$-induced caused hypertension and increased organs weight that may cause early process of damages in organs.
\end{abstract}

\section{Introduction}

High blood pressure (BP) is a very common disorder, and its complications (including heart disease, stroke, and kidney disease) are major public health problems. ${ }^{1}$ Among the environmental factors that affect blood pressure, dietary sodium chloride has been studied the most, and there is general consensus that increased sodium chloride intake increases blood pressure.

High salt intake promotes the elevation of blood pressure, cardiac hypertrophy, the impairment of left ventricular relaxation, endothelial dysfunction, and kidney injury. ${ }^{1}$ Previous study in experimental animal models of salt-sensitive hypertension demonstrated that immune systems played a role in mechanism of hemodynamic in kidney. $^{2}$ Renal tubule interstitial vascular inflammation is associated with salt-sensitive models of hypertension, and more evidence shows that immunosuppressive therapy prevents the development of hypertension by reducing $\mathrm{T}$ lymphocytes in the kidney. ${ }^{3}$

The role of $\mathrm{NaCl}$ is supported by insight into the mechanism of pressure-natriuretic, ${ }^{1}$ monogenic forms of hypertension, 4 and studies of food salt reduction. ${ }^{5}$ Autoregulation disorders induced by $\mathrm{NaCl}$ can conspire with adverse central hemodynamics to promote accelerated kidney function. ${ }^{5}$ Recent epidemiological studies have shown an association of serum $\mathrm{Cl}^{-}$and $\mathrm{Na}^{+}$on mortality. ${ }^{6}$ In usual diets, more than $85 \%$ of $\mathrm{Na}^{+}$ is consumed as sodium chloride, and clinical evidence showed that the independent effect of $\mathrm{Cl}^{-}$on blood pressure and prognosis has been considered. ${ }^{1}$

Extensive investigations in several models of hypertensive rats and humans show that loading with equimolar amounts of sodium salts causes similar degrees of $\mathrm{Na}^{+}$retention, weight gain, and suppression of RAAS. $\mathrm{NaCl}$ may also potentiate the effects of adverse central hemodynamics on the heart. In isolated perfused kidneys, and in kidneys perfused in situ, hyperchloremia results in renal vasoconstriction and a decline in glomerular filtration rate as a consequence of tubuloglomerular feedback. ${ }^{7}$

One important contributor to hypertension in saltsensitive animal models and humans seems to be the endothelial dysfunction, in particular the altered vascular reactivity due to an impairment in nitric oxide (NO) production. Apart from the effects on BP regulation, elevated salt intake was associated with cardiovascular and renal changes leading to end organ damage. ${ }^{5}$ However, this study was to examine blood pressure and organs weight changes in high salt diet in mice. 


\section{Methods}

\section{Animal}

Eight weeks old male Mus musculus were purchased from Animal Laboratories (Universitas Airlangga). The animals were acclimatized at room temperature (25$30^{\circ} \mathrm{C}$ ) for one week; and had access to food and water ad libitum under a 12-h light/12-h dark. After 1 week of acclimation, the mice were given a high- $\mathrm{NaCl} 8 \%$ by intraperitoneal for 8 hours.

\section{Blood Pressure Measurement}

The onset and development of hypertension were assessed by using the tail-cuff plethysmography method blood pressure system (IITC Life Science). The average of five pressure readings was recorded for each measurement.

\section{Statistical Analysis}

All results were expressed as mean \pm SEM. The significance between two groups was performed with unpaired student's t-test with SPSS ver. 25. $\mathrm{P}<0.05$ was considered statistically significant.

\section{Results}

\section{NaCl-induced increased blood pressure}

Our initial studies evaluated the biological effects of giving a mildly hypertonic solution of salt to mice in short time. We first evaluated the effects of salt intake in blood pressure after 8 hours administration of $\mathrm{NaCl}$ by oral gavage. To evaluate the effect of salt intake on blood pressure, mean arterial pressure was measured in animals with $8 \% \mathrm{NaCl}$ administration compared with control group $(\mathrm{p}<0.05)$. (Figure 1)

\section{$\mathrm{NaCl}$-induced increased weight of organs}

To examine whether $\mathrm{NaCl}$-induced improved organ damages, the weight of organ was measured. Figure 2 showed that $\mathrm{NaCl}$ administration increased the weight of heart and kidney significantly compared with control group.
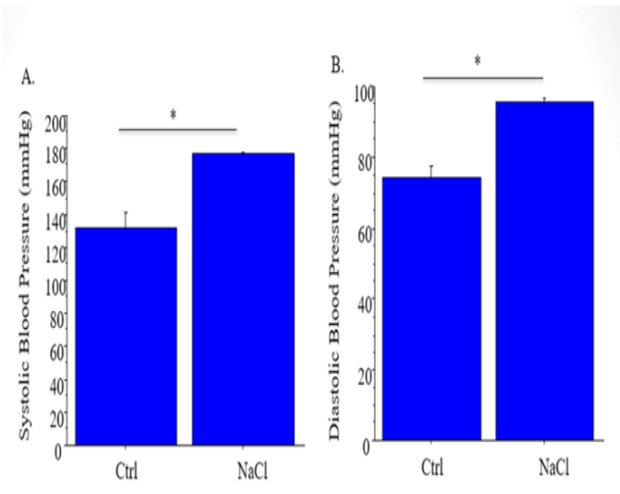

Figure 1. NaCl-induced high blood pressure (A) $\mathrm{NaCl}$ administration increased systolic blood pressure and (B) diastolic blood pressure compared with control group (n; 10 per group). *; $\mathrm{P}<0.05$. All values were mean \pm SEM.
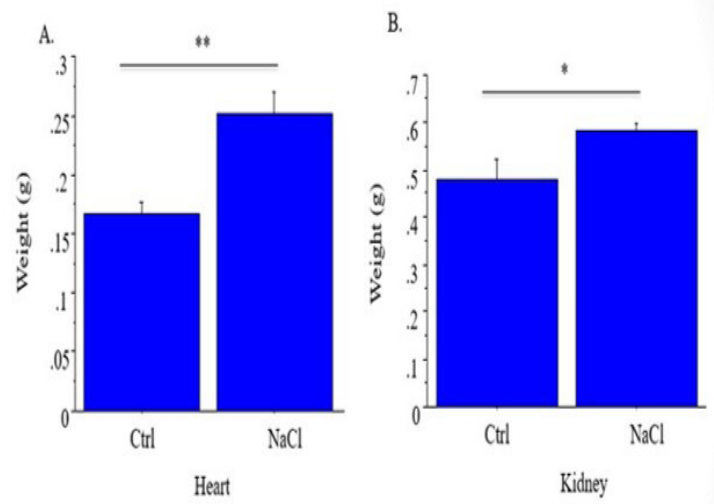

Figure 2. NaCl-induced high blood pressure (A) $\mathrm{NaCl}$ administration increased the weight of heart and (B) kidney compared with control group ( $\mathrm{n} ; 10$ per group). *; $\mathrm{P}<0.05$; $* * \mathrm{P}<0.01$. All values were mean $\pm \mathrm{SEM}$.

\section{Discussion}

Salt is an essential micronutrient with no calorie, however it has been associated with development of metabolic syndrome such as hypertension, diabetes mellitus, and obesity. Some previous studies have shown that sodium chloride $(\mathrm{NaCl})$ intake is directly related to increase blood pressure and increase cardiovascular risk. In this study we investigated the effect of short-term $\mathrm{NaCl}$-induced hypertension and organ damages. Evidence in experimental animals demonstrated that salt sensitivity of arterial pressure in many animal species, including primates. ${ }^{8}$

In present study, we found that short-term administration of high salt significantly increased systolic and diastolic blood pressure. The potential for increased dietary salt to influence blood pressure has been recognized and studied for decades. Blood pressure is salt-sensitive in almost 26 million Americans, and many of the mechanisms that contribute to salt-sensitive hypertension have been reviewed.9 Mattson, et al. recently published a series of studies demonstrating an important role for the adaptive immune system in the development of salt-sensitive hypertension in rats. ${ }^{10}$

In addition to increased arterial pressure, excessive salt intake is linked to cardiovascular and renal injury. ${ }^{11}$ However, in the present study we found that administration of high salt in short-term significantly increased the organs size, especially in heart and kidney compared with control group. These results indicated that the administration of a high salt diet in a short time could cause changes in organs, which indicated that vascular damage had begun to occur in the organs that maintained the balance of blood pressure in the heart and kidneys. Previous study already explained that level measured by augmented ratios of heart weight and body weight with high level of BP had correlation with extent of cardiac hypertrophy. ${ }^{12}$

Increased arterial pressure causes target organ injury; consequently, salt-induced cardiovascular and renal injury may be related to concomitant increase in arterial pressure. However, there is a plethora of evidence from animal and clinical studies demonstrating that in addition to this arterial pressure--mediated effect, there are direct adverse effects on cardiovascular and renal function and structure. ${ }^{9}$ 
The critical role of kidney dysfunction in salt-induced increases in BP in sensitive subjects is highlighted by the fact that the most reproducible and consistent forms of experimental salt-sensitive hypertension are induced by impairing kidney function in various ways that reduce glomerular filtration rate (GFR) or increase tubular reabsorption. Also, all known monogenic forms of salt-sensitive human hypertension are characterized by mutations that directly or indirectly increase renal $\mathrm{NaCl}$ reabsorption. ${ }^{13}$

\section{Conclusion}

High NaCl-induced intake increased systolic and diastolic blood pressure and the weight of kidney and heart. This results may have correlation with early stage of organs damages.

\section{Conflict of Interest}

The author stated there is no conflict of interest

\section{References}

1. Kaplan NM. Kaplan's clinical hypertension. Lippincott Williams \& Wilkins, 2010

2. De Miguel C, Das S, Lund H and Mattson DL. T lymphocytes mediate hypertension and kidney damage in Dahl salt-sensitive rats. American journal of physiology Regulatory, integrative and comparative physiology. 2010; 298: R1136-42.

3. Kotchen TA, Cowley AW, Jr. and Frohlich ED. Salt in health and disease--a delicate balance. The New England journal of medicine. 2013; 368: 1229-37.

4. Cook NR, Cutler JA, Obarzanek E, et al. Long term effects of dietary sodium reduction on cardiovascular disease outcomes: observational follow-up of the trials of hypertension prevention (TOHP). Bmj. 2007; 334: 885-8.
5. Hall JE. Renal Dysfunction, Rather Than Nonrenal Vascular Dysfunction, Mediates Salt-Induced Hypertension. Circulation. 2016; 133: 894-906.

6. McCallum L, Jeemon P, Hastie CE, et al. Serum Chloride Is an Independent Predictor of Mortality in Hypertensive Patients. Hypertension. 2013; 62: 836-43.

7. Schmidlin O, Tanaka M, Sebastian A and Morris RC, Jr. Selective chloride loading is pressor in the stroke-prone spontaneously hypertensive rat despite hydrochlorothiazide-induced natriuresis. Journal of hypertension. 2010; 28: 87-94.

8. Whelton PK, Carey RM, Aronow WS, et al. 2017 ACC/AHA/ $\mathrm{AAPA} / \mathrm{ABC} / \mathrm{ACPM} / \mathrm{AGS} / \mathrm{APhA} / \mathrm{ASH} / \mathrm{ASPC} / \mathrm{NMA} / \mathrm{PCNA}$ Guideline for the Prevention, Detection, Evaluation, and Management of High Blood Pressure in Adults: A Report of the American College of Cardiology/American Heart Association Task Force on Clinical Practice Guidelines. Hypertension. 2018; 71: e13-e115.

9. Sanada H, Jones JE and Jose PA. Genetics of salt-sensitive hypertension. Current hypertension reports. 2011; 13: 55-66.

10. Mattson DL, James L, Berdan EA and Meister CJ. Immune Suppression Attenuates Hypertension and Renal Disease in the Dahl Salt-Sensitive Rat. Hypertension. 2006; 48: 149-56.

11. Walkowska A, Kuczeriszka M, Sadowski J, et al. High salt intake increases blood pressure in normal rats: putative role of 20-HETE and no evidence on changes in renal vascular reactivity. Kidney \& blood pressure research. 2015; 40: 323-34.

12. Crowley SD, Gurley SB, Herrera MJ, et al. Angiotensin II causes hypertension and cardiac hypertrophy through its receptors in the kidney. Proceedings of the National Academy of Sciences of the United States of America. 2006; 103: 17985-90.

13. Rossier BC, Staub O and Hummler E. Genetic dissection of sodium and potassium transport along the aldosterone-sensitive distal nephron: importance in the control of blood pressure and hypertension. FEBS letters. 2013; 587: 1929-41. 